\title{
El pensamiento posmoderno en los discursos sobre la práctica del diseño
}

\author{
Mariana Pittaluga \\ Universidad Nacional de La Plata \\ Recibido: 2 de julio del 2018 / Aprobado: 16 de agosto del 2018 \\ doi: 10.26439/limaq2019.n005.4526
}

Este trabajo intenta poner de manifiesto la influencia de los discursos de la posmodernidad en los nuevos discursos sobre la práctica del diseño. Asumimos que con la crisis de la modernidad se resquebraja también el corpus de discursos sobre su práctica del diseño - entendido como heredero suyo-, lo que da lugar a nuevas posturas y argumentaciones que, a su vez, modifican la propia práctica y determinan la expansión de su campo. Partiendo del campo filosófico para comprender el debate modernidad-posmodernidad, analizamos sus influencias y matices en los autores más importantes en el ámbito del diseño.

posmodernidad, modernidad, diseño, discursos de la práctica de diseño

\section{Postmodern thinking in discourses on the practice of design}

This paper aims to demonstrate the influence of postmodern discourses on new discourses on the practice of design. By understanding the design as a product of modernity, we assume that the crisis of this cultural construction also breaks down the corpus of discourses on the practice of design, giving rise to new positions and arguments which, at the same time, modify the practice itself and determine the expansion of its field. Taking philosophy as a starting point to understand the debate on modernity-postmodernity, we analyze its influences and shades according to the most influential authors in the field of design.

postmodernity, modernity, design, discourses on the practice of design 


\section{INTRODUCCIÓN}

Bajo los metarrelatos del pensamiento moderno (Lyotard,1986/1994) fueron fundadas las bases del diseño, su delimitación y definición (Devalle, 2009a).De este modo, se consagra como un campo (Bourdieu, 2005) autónomo dentro de las prácticas culturales, con un habitus y capital simbólico compartidos, y, por tanto, deslindado del campo artístico, tecnológico y científico. Esta autonomía se debe en gran parte a su componente diferencial: lo proyectual (Maldonado, 1977/1993), que define la especificidad del diseño a través de este saber instrumental, fruto de un pensamiento racional. En esto último - el ser proyectual o ser artístico- consistió el debate moderno puertas adentro del diseño. En definitiva, las preocupaciones de su discurso giraban en torno a una pregunta: ¿qué es el diseño?

La racionalidad es central en lo que Maldonado define como proyectual; por tanto, si, desde una perspectiva moderna, lo proyectual es esencial para la práctica del diseño y la racionalidad en que se sustenta es lo que la posmodernidad pone en crisis, no es desatinado pensar que habrá transformaciones en lo que respecta a la práctica del diseño y a sus discursos ${ }^{1}$. En este sentido, advertimos en algunas indagaciones actuales sobre la práctica del diseño que las preguntas no giran en torno a este como disciplina o a su producto como objeto de estudio, sino más bien se centran en la operatoria del diseño para resolver problemáticas dentro de un contexto complejo (Morin, 1990/1997). La pregunta entonces sería esta: ¿cómo opera el diseño en y para una determinada situación?

Creemos que este cambio de eje en las indagaciones sobre el discurso del diseño y el particular interés por su práctica - hechas por Richard Buchanan, Kees Dorst, Guy Julier, Victor Margolin, Abraham Moles, Harold Nelson, Erik Stolterman, John Thackara, entre otros - solo es posible ser pensado dentro de un discurso que trascienda los preceptos de la modernidad,

1 Debemos aclarar que no se trata de una transformación generalizada, sino que simultáneamente circulan discursos que se circunscriben al pensamiento moderno (G. Bonsiepe, J. Frascara, J. Costa, entre otros). 
sobre todo de su énfasis en la razón como un dominio hegemónico y de pretensión totalizante.

Por último, y más importante aún, si efectivamente hay una influencia del pensamiento posmoderno en el discurso del diseño, consecuente e indefectiblemente se reconfigura su campo. Es de nuestro interés en estas páginas dejar esbozado un camino posible para desarrollar los alcances, los modos y las consecuencias de esta reconfiguración.

\section{EL DEBATE MODERNIDAD-POSMODERNIDAD}

Cuando hablamos de posmodernidad, lo hacemos en el sentido de la caracterización propuesta por J. F. Lyotard en su texto La condición posmoderna (1979/1993), aunque no es la única idea que se tiene sobre el tema, dado que en textos elaborados por Huyssen, Vattimo, Foster, Jameson, entre otros, también está presente el tema. Esto destaca la calidad no unidimensional de los discursos sobre la posmodernidad.

A diferencia de la era moderna, que nació con el establecimiento de la subjetividad en el discurso, se caracteriza por la competencia constante entre una multiplicidad de juegos del lenguaje, sin que alguno se postule como la forma legítima de dar cuenta de la realidad. De este modo, a partir de la deslegitimación de la racionalidad, postula el fin de la historia, revelando que, en esta última, la razón solo ha sido una entre otras narrativas, un gran relato al cual renuncia.

En lugar de la creencia en un gran relato, se insta a la relevancia del carácter irreductible y pluralista de los discursos. En este sentido, surge el interés por los fragmentos, es decir, ya no se trata de un discurso racional y totalizante en singular, sino de una multiplicidad de discursos en plural. No hay más lugar para las ideas de consenso (Habermas), historia (Hegel) o progreso (Ilustración).

Para la posmodernidad son bienvenidos el azar, el desorden de los procesos naturales y el principio de incertidumbre de Heisenberg, y aunque hay debates que se centran en que de alguna manera han sido malinterpretados 
—especialmente por el arte-, lo destacable es que hay una apertura hacia nuevos horizontes epistemológicos.

Se establece, entonces, una scienza nuova contrapuesta a los preceptos reduccionistas y simplificantes de la ciencia moderna, y fundada casi a través de sus antónimos: unidad versus diversidad, azar versus necesidad, cantidad versus calidad, sujeto versus objeto, holismo versus reduccionismo. Como indica Morin (1990/1997), "porque es, evidentemente, toda la estructura del sistema de pensamiento la que se halla trastornada, transformada, es toda una enorme estructura de ideas la que colapsa".

Esta ciencia nueva es la que permite pensar la complejidad, que, como expresa Morin, no se plantea solo en el ámbito de la ciencia, sino que atraviesa también el de la vida cotidiana. Su teoría de la complejidad está fundada firmemente en la renuncia a los imperativos modernos. Con ello, da un giro a los "demonios" científicos y filosóficos, y los convierte en la fortaleza del paradigma complejo.

Fundamentalmente, a partir de las dudas planteadas por Nietzsche, las corrientes posmodernas surgen de la deslegitimación de los relatos modernos y la crítica a una ideología totalizadora. De esta manera se abandona aquel proyecto moderno aferrado a las ideas ilustradas de un discurso universal, racional y científico.

¿Dónde puede residir la legitimación después de los meta-relatos? El criterio de operatividad es tecnológico, no es pertinente para juzgar lo verdadero y lo justo. ¿El consenso obtenido por discusión, como piensa Habermas? Violenta la heterogeneidad de los juegos del lenguaje.Y la invención siempre se hace en el disentimiento. El saber postmoderno no es solamente el instrumento de los poderes. Hace más útil nuestra sensibilidad ante las diferencias y fortalece nuestra capacidad de soportar lo inconmensurable. (Lyotard, 2008, pp. 4-5)

El pensamiento de Habermas, caballo de batalla por antonomasia de los defensores del proyecto moderno, plantea una defensa en contraposición al pensamiento posmoderno que caracteriza como antimoderno.

La célebre conferencia de 1980 de la Bienal de Venecia inaugura uno de los momentos más álgidos en el debate modernidad-posmodernidad, debido a la repercusión que tuvo entre los representantes del cuestionamiento a los 
imperativos modernos —uno de sus más importantes interlocutores fue Lyotard-.

Es preciso señalar que la defensa de Habermas es de la modernidad que propuso un proyecto emancipatorio y de liberación de la humanidad respecto del pasado, es del proyecto de la Ilustración, que, según su argumentación, no se trata de un proyecto fracasado sino inacabado. De allí la palabra proyecto.

Este proyecto es el que el filósofo alemán opone a los modernismos estéticos - se refiere particularmente al dadaísmo y surrealismo- y es en este sentido que sentencia que "el modernismo es dominante, pero está muerto" (Habermas, 2008, p. 24).

Habermas defiende el proyecto moderno a través de la crítica de las posiciones adversas. En primer lugar, encara los juicios contra lo que interpreta como antimodernismo, representado por el neoconservadurismo. En este sentido, traza una conexión entre la posmodernidad y la posición neoconservadora. Encuentra que los argumentos neoconservadores - y principalmente se refiere a la obra de Bell — hacen un análisis débil o ingenuo de la modernidad cuando escinden la modernización social del desarrollo cultural, y olvidan que tanto lo que rescatan como lo que descartan se gesta en el mismo seno.

Del mismo modo, critica que la única solución que plantea el neoconservadurismo para combatir las desviaciones hacia el consumo, el éxito y el ocio sea retomar un discurso religioso y desconocer, de este modo, las lógicas propias de la modernidad.

En última instancia, el neoconservadurismo, sobre la base de las actitudes modernistas, justifica un antimodernismo. Toma de la modernidad lo que cree conveniente - como el desarrollo de la ciencia-, pero deja confinado a la reprobación lo que no cuaja en su postura. Habermas advierte que, detrás de este razonamiento, la ciencia, la moralidad y el arte se entienden como estratos autónomos, lo que es lo mismo que abandonar el proyecto de la modernidad, ya que tomar algunos recursos y otros no implica no entender su lógica.

Si bien esta crítica al neoconservadurismo es transparente en su escrito, los críticos de Habermas, principalmente Lyotard, han señalado que el foco de su ataque se centra en el posmodernismo, desde el momento en que articula 
el pensamiento posmoderno francés con el neoconservadurismo, en tanto la renuncia a la racionalidad de los primeros allana el camino de los segundos.

En respuesta a esta crítica habermasiana, Lyotard plantea lo siguiente:

Lo que Habermas reclama a las artes y a la experiencia que éstas procuran es, en suma, que sean capaces de tender un puente por encima del abismo que separa el discurso del conocimiento del discurso de la ética y la política, franqueando así un pasaje hacia la unidad de la experiencia.

La pregunta que yo planteo es la siguiente: ¿a qué tipo de unidad aspira Habermas? ¿El fin que prevé el proyecto moderno es acaso constitución de una unidad sociocultural en el seno de la cual todos los elementos de la vida cotidiana y del pensamiento vendrían a encontrar su lugar como un todo orgánico? ¿O es que el pasaje que se ha de franquear entre los juegos de lenguaje heterogéneos, el conocimiento, la ética, la política, es de un orden diferente de éstos? Si es así, ¿cómo haría para realizar su síntesis efectiva? (1986/1994, p. 13)

Así, lo denominado posmodernidad supone la pluralidad, la multiplicidad, la contradicción, la simultaneidad, en lugar de la adscripción moderna al progreso unilineal y a la univocidad. El momento posmoderno, en términos poshistóricos, congenia con las ideas de fragmento y fractura, así como con el compromiso con las minorías.

Este giro de pensamiento hacia la fragmentación y el pluralismo - como producto de los aportes de Nietzsche y Wittgenstein en relación con la destrucción de la unidad del lenguaje - ha repercutido de varias formas en las artes, la arquitectura y, según indagaremos, en el diseño.

Estos cambios han sido prefigurados por las vanguardias históricas, influenciadas por el pensamiento nietszchiano, y desarrollados en los discursos sobre la práctica del diseño. Sin embargo, han sido cercenados por el discurso dominante de la modernidad. Recordemos, sin ir más lejos, el famoso debate enmarcado por la sentencia de A. Loos en 1908, "el ornamento es delito", en pleno auge de la legitimación de la racionalidad como eje del discurso moderno del diseño y motor de su praxis.

Los debates en torno a modernidad-posmodernidad que entablan Habermas y Lyotard son centrales para nuestro trabajo, puesto que mediante ellos 
entendemos que el diseño, como práctica cultural, prefigura y transforma su discurso como consecuencia directa de la reconfiguración de su campo.

De este modo, entendemos que, en la instalación del debate entre estas dos perspectivas de pensamiento, se consolida un punto de inflexión para la construcción del discurso del diseño, lo que abre las puertas hacia nuevos horizontes en las indagaciones sobre el tema, surcadas por la tensión entre la defensa y el cuestionamiento al discurso de la modernidad.

\section{¿DISEÑO Y POSMODERNIDAD? CRÍTICAS AL DISCURSO MODERNO DEL DISEÑO}

La crítica a la modernidad, y específicamente al Movimiento Moderno, en los discursos sobre la práctica del diseño aparece enfocada, en primer lugar, en el aspecto funcionalista. El styling, el kitsch del siglo XIX y el modern style fueron algunas de las respuestas contemporáneas al funcionalismo institucionalizado por la Bauhaus.

Esto evidencia que el debate forma-función instalado por la Bauhaus, si bien se convirtió en el modo de hacer dominante para el diseño occidental, nunca dejó de verse amenazado por contradiscursos emergentes, puesto que sus propias contradicciones conformaban su talón de Aquiles. Como así señalara J. Baudrillard, "basta llevar a fondo este principio de funcionalidad para hacer que surja de él el absurdo" (2008, p. 235).

No obstante, a partir de la segunda mitad del siglo xx, el debate forma-función pierde vigencia ante las grandes transformaciones que se producen en la sociedad de consumo, cuando se subvierte la lógica de oferta y demanda (Devalle, 2009b).

Su batalla (la del funcionalismo) cristalizó a partir de estos principios: eliminar sistemáticamente lo inútil y, por este mismo hecho, determinar una filosofia de la vida. Por lo tanto, no existen ya objetos, cualesquiera sean, que no pretendan, en cualquier medida, participar de alguna manera de la inspiración de lo funcional, aun cuando la nieguen o la contradigan.

Esta tesis entra en contradicción, de hecho, con las ideas de una sociedad opulenta. [...] En otros términos, el mercado está preso en una circulación 
que necesariamente debe ir en aumento. Se trata de una ética de lo superfluo, de lo perentorio incorporado y del consumo a ultranza. La ética del consumo es, como bien señala Baudrillard, antiascética, pues el ascetismo de la función construye el objeto para una razonable eternidad. (Moles, 1971/1973, p. 175)

Con la transformación de las condiciones del mercado, se debilita el argumento racionalista, que estaba determinado por una economía que, en términos de Baudrillard, funcionaba a partir del valor de uso y de cambio. A diferencia de este nuevo contexto, la transformación económica traerá consigo la primacía del valor simbólico. Esto, sumado a la instalación de la desconfianza en los pilares modernos, desemboca en lo que se podría denominar la crisis de la modernidad.

Antes de seguir avanzando, es preciso aclarar que así como Maldonado ha marcado la diferencia entre modernidad y Movimiento Moderno, la misma salvedad debemos hacer respecto al término posmodernidad y lo que algunos llaman estilo o diseño posmoderno. Al primero lo inscribimos dentro de la esfera filosófica, como hemos planteado basándonos principalmente en Lyotard. Se trata de una corriente de pensamiento — sin un discurso homogéneo, sino multidimensional - que cuestiona los imperativos de la modernidad.

En cambio, lo que comúnmente se expresa como diseño posmoderno está relacionado con los lenguajes estéticos que surgen como contrapartida del estilo moderno. La arquitectura deconstructivista, el grunge, los diseños del Memphis Group, Alessi y Arad, entre muchos otros, forman parte de esos lenguajes.

Nuestro enfoque no está dirigido hacia esa esfera, sino que busca las relaciones, si es que las hubiere, entre los discursos actuales sobre la práctica del diseño y algunos ejes del vasto pensamiento posmoderno.

Como hemos visto, el discurso moderno estaba fundado en las ideas de progreso, al cual se llegaba a través de un proceso lineal que indefectiblemente trataba de la superación del presente. El discurso moderno era utópico y el diseño hizo eco de él. La crisis de la modernidad, basada en la renuncia a estos imperativos, significa el desplome de la utopía, de la fe en el progreso y, con ello, la desconfianza frente a todas las prácticas que se habían albergado en los preceptos modernos. 
El sentido de permanencia anhelado por la modernidad se convertirá en el principio de lo efimero de la posmodernidad (Pelta, 2004).

\section{Nuevos planteos}

En virtud de ubicar y describir las trazas del pensamiento posmoderno en los discursos sobre la práctica del diseño, analizaremos un conjunto de textos de autores diversos y sus respectivas vinculaciones con los aspectos filosóficos de la posmodernidad y la noción de complejidad. En función de la limitada extensión de este trabajo frente a la minuciosidad de muchos de esos textos, creemos importante advertir que nos vemos limitados a presentar recortes muy parciales que convienen a nuestro objeto de estudio. A fin de no traicionar lo expresado por los diferentes autores, se ha recurrido, en algunos casos, a la cita extensa aun cuando no dé cuenta del contexto total.

Uno de los autores contemporáneos de más influencia en el discurso del diseño actual es el estadounidense Richard Buchanan, cuyos aportes se centran en el rol del diseño y el diseñador en un nuevo contexto. Para este autor, el diseño es un elemento fundamental de una nueva filosofia de la cultura.

Una de las ideas fuertes de su pensamiento es la del argumento retórico del diseño. Esta concepción — que postula que el diseñador, en lugar de hacer objetos, en realidad construye un argumento - dista de una idea moderna del diseño, donde la preocupación — con todo lo que conllevaes la construcción de objetos. Como indica Buchanan (1985/1989), "el diseñador, en vez de simplemente hacer un objeto o cosa, está en realidad creando un argumento persuasivo que cobra vida cuando un usuario considera o utiliza un producto como un medio para cierto fin" [traducción de la autora] (pp. 95-96)².

Por otro lado, Buchanan (1985/1989) describe a la posmodernidad como "un período de desilusión posterior al exceso de confianza en el brillante futuro ofrecido por la ciencia y la tecnología” (p. 96), y bajo estas circunstancias el

2 "This article suggests that the designer, instead of simply making an object or thing, is actually creating a persuasive argument that comes to life whenever a user considers or uses a product as a means to some end". 
diseño ha transitado diversos caminos que conviven simultáneamente. En tal sentido, dice:

En todos estos casos, sin embargo, el diseño es un debate entre puntos de vista opuestos sobre aspectos como la tecnología, la vida práctica, el lugar de la emoción y la expresión en el ambiente de vida; y el anfitrión de otras preocupaciones que conforman la textura de la vida posmoderna, posindustrial [traducción de la autora]. (p. 96) ${ }^{3}$

Buchanan retoma la idea de Harold Rosenberg al afirmar que el diseño se declara a sí mismo, es decir, que los objetos diseñados declaran un estatus mediante una argumentación persuasiva. Como ejemplo, cita los artefactos de la marca Braun: el diseñador utiliza estrategias para dotar de una aparente simplicidad a sistemas altamente complejos y lograr que se presenten como objetos "amigables". Esa simulada simplicidad, que en realidad no es tal, pero que el usuario no llega a percibir como artificial, es en sí un discurso retórico.

La preocupación de Buchanan por el lugar del diseño en la posmodernidad se refleja también en su exposición crítica del dilema de Branzi en su artículo "Branzi’s dilemma: design in contemporary culture" (1994/2010).

Las circunstancias en las cuales Branzi desarrolla su pensamiento están arraigadas en el colapso del modernismo. Los ideales modernistas, que invocaban el continuo mejoramiento de la condición humana a través del progreso en el arte, el diseño y la tecnología, resultan insolventes como aglutinador ideológico del diseño y la cultura. "El paracaídas ideológico del modernismo ya no funciona”, dice Branzi (como se citó en Buchanan, 1994/2010).Y frente a esta ausencia de identidad colectiva plantea dos alternativas: la formulación de una visión por cuenta de cada individuo o la de una nueva ideología que sustituya a la modernidad bajo la forma de un "nuevo modernismo".

Cualquiera de estas opciones, dice Buchanan, son desagradables y peligrosas. La primera puede conducir a un relativismo que en última instancia suprime horizontes alternativos, y la segunda conlleva la pregunta de cuál ideología

3 "In all of the cases, however, design is a debate among opposing views about such matters as technology, practical life, the place of emotion and expression in the living environment, and a host of other concerns that make up the texture of postmodern, postindustrial living". 
debería ser esa. Buchanan (1994/2010) plantea que esta dicotomía dialéctica no puede ser tal: "a algunos autores les encanta presentar dos columnas paralelas de términos, 'modernos' de un lado y 'posmodernos' del otro, como si la historia ofreciera una elección clara entre un término y el otro" [traducción de la autora] (p. 24) .

A diferencia de Branzi, Buchanan plantea una tercer alternativa: partiendo del concepto - hoy muy en boga- de design thinking, propone que en un periodo donde los valores de usabilidad y disfrute a menudo parecen irreconciliables, los diseñadores pueden ofrecer ejemplos de nuevas maneras de pensamiento y hacer.

Hay esperanza en que el design thinking, aplicado en nuevas áreas, pueda servir como alternativa a las viejas formas de tecnología basadas en la especialización científica, las que expertos en áreas estrechas del conocimiento una vez creyeron que podrían mejorar, y enriquecer la vida aplicando conocimiento científico para resolver los problemas cotidianos. [...] La tarea del diseño no es la de diseñar para una audiencia universal o grupos nacionales o segmentos del mercado, ni siquiera para "el consumidor". A pesar del continuo rol de la producción en masa en muchas sociedades, la tarea del diseño es diseñar para el individuo situado en su contexto inmediato. Nuestros productos deben sustentar al individuo en su esfuerzo por convertirse en partícipe activo de la cultura. $(1994 / 2010, \text { p. } 25)^{5}$

Cabe aquí resaltar el concepto de cultura en Buchanan, quien critica también a Branzi por su visión de la cultura como "una ideología", y, por tanto, excluyente. Para Buchanan, hay que hablar de "culturas" en interacción

4 "Some writers delight in the exercise of presenting two parallel columns of terms, with 'modernist' terms on one side and opposing 'postmodernist' terms on the other side, as if history or intelligence offered a clear choice between one term or the other".

5 "There is hope that design thinking, applied in many new areas, can serve as an alternative to the old forms of technocracy based on scientific specialization, where experts in narrow areas of learning once believed that they could improve and enrich life merely by applying technical knowledge to solve the problems of everyday life. [...] The task is no longer to design for a universal audience, or national groups, or market segments, or even the ideological abstractions known as 'the consumer'. Despite the continuing role of mass production in many societies, the task is to design for the individual placed in his or her immediate context. Our products should support the individual in the effort to become an active participant in culture". 
y debate. Esta postura, marcadamente posmoderna, se pone también en evidencia en su interés por los design studies (estudios de diseño) para el abordaje de la práctica del diseño. Esta disciplina surge como mediadora entre los problemas del diseño integrador en la industria y las preguntas de siempre sobre el rol y las contribuciones del diseño.

Los design studies hacen explícita la diversidad de los presupuestos que guían al diseño y examinan sus consecuencias en el pasado, presente y futuro. Están dirigidos a mejorar la práctica del diseño, pero desde una perspectiva pluralista y de diferentes abordajes teóricos. Esto indica una realidad sobre la práctica del diseño, que implica un distanciamiento de aquellos preceptos monolíticos sobre ella. En palabras del mismo autor: "el diseño es demasiado importante para confinarlo a preguntas y soluciones tácticas" [traducción de la autora] (Buchanan, 1989) ${ }^{6}$.

Dentro de esta concepción del diseño — que en principio podemos llamar expandida y sobre la cual volveremos en nuestras conclusiones-, aparece también en Buchanan la inclusión de los procesos inmateriales en el campo del diseño. Como lo expresa G. Julier:

Buchanan no especifica necesariamente qué entiende por "productos", y afirma que estos pueden englobar tanto símbolos comunicativos e imágenes como objetos físicos; pero también considera la función del diseño en la configuración de sistemas, entornos, ideas y valores. En este estado de madurez, el diseño puede participar en la presentación externa de bienes y servicios ante el público, pero también de los sistemas internos que administran el desarrollo y la distribución de esos bienes. Así pues, traslada el debate de la forma material a los procesos inmateriales, del diseño como proveedor de objetos al modelado de relaciones y estructuras. (Julier, 2008/2010, p. 76)

Este descentramiento respecto de la producción objetual y el interés por los aspectos inmateriales del diseño encuentra en Abraham Moles un exponente clave. Es notable la idea planteada por Moles (1995) en su ensayo "Design and inmmateriality: what of it in a post-industrial society?", publicado en el libro The idea of design, editado por R. Buchanan, D. Doordan y V. Margolin.

6 "Design is too important to be confined to tactical questions and tactical solutions". 
Allí plantea que una cultura inmaterial está emergiendo como consecuencia de cambios tecnológicos. Sostiene que la sociedad posindustrial, superindustrializada, se encuentra en la era de la telepresencia, que procura establecer equivalencias entre la presencia real y la presencia vicarial. Este último aspecto realza la importancia de lo que se encuentra próximo en contraposición con lo distante, pero, al mismo tiempo, que la distancia entre nosotros y los objetos se vuelve cada vez más irrelevante. Por otro lado, en la sociedad de la información por la que transitamos, pasamos más tiempo manipulando información que objetos.

El diseñador crea el entorno de otros. Hasta ahora, la vocación del diseñador ha sido tanto conceptual como concreta. El taller ha sido un lugar para percibir y construir modelos destinados a ser copiados para la producción masiva. La pregunta ahora, sin embargo, es cómo esta vocación se modificó, para bien o para mal, por el inexorable desarrollo de la cultura inmaterial. La actividad misma del diseño está cambiando porque las herramientas del diseñador se están volviendo inmateriales como las vidas de aquellos para quienes los productos se comercializan.

[...] La tendencia hacia la inmaterialidad incluye toda la concepción proyectual en un modelo concreto, un proceso que solía depender de una situación de interacción permanente entre concepción y construcción. El juego dialéctico entre lo abstracto (la idea, la visión mental) y lo concreto (la lucha con el material, herramientas y dispositivos) está dejando paso a un trabajo hecho esencialmente con computadoras de escritorio integradas a las de la fabricación [traducción de la autora]. (Moles, 1995, p. 270) ${ }^{7}$

7 "The designer creates the environment of others. Until now the designer's vocation has been both conceptual and concrete. The workshop has been a place to perceive and build models destined to be copied for mass production. The question now, however, is how this vocation has been modified, for the better or for the worse, by the inexorable development of the immaterial culture. The design activity itself is changing because the designer's tools are becoming immaterial, as are the lives of those to whom the products are marketed. [...] The trend toward immaterialism includes all projectional conception in a concrete model, a process which used to depend on a situation of permanent interaction between conception and construction. The dialetic game between the abstract (the idea, the mental vision) and the concrete (the struggle with the material and disparate tools and appliances) is giving way to work done essentially with computer-integrated manufacturing at computer desk". 
Antes de introducir algo nuevo, el diseñador debe proteger el status quo, el cual permite a los individuos participar espontáneamente y con poco esfuerzo en la seductora inmaterialidad del mundo de hoy [traducción de la autora]. $\left(\right.$ Moles, 1995 , p. 271) ${ }^{8}$

Este nuevo aspecto de la cultura que señala Moles como inmaterial define una postura que determina un cambio indefectible en la práctica del diseño. Del mismo modo, entendemos que este planteo necesariamente se posiciona un paso más allá del discurso moderno.

Ampliando el horizonte del diseño desde el sentido en que son comprendidos los objetos y su relación con el usuario, aunque sin apartarse tanto de los preceptos tradicionales como Buchanan o Moles, encontramos a Victor Margolin. En su artículo "Expanding bounderies of design: the product enviroment and the new user" (1989), plantea que los objetos están comprendidos per se y por su entorno como totalidad. Esto incluye las condiciones para la adquisición del producto, aprender a usarlo, seguir sus cambios y mejoras, obtener sus componentes y mantenerlo en buen estado. De este modo, aunque no fuera así considerado por los diseñadores y fabricantes, para el usuario el producto es también su entorno.

Este planteo de la relación entre producto y usuario está un paso adelante de la noción de interfase de Bonsiepe. El objeto diseñado no es solamente lo que relaciona al usuario con un fin —en el que el diseñador preveía un número limitado de funciones-, sino que la noción de Margolin supone que los objetos diseñados deberán ser pensados como dinámicos en contraposición con la forma convencional estática, como resultado del proceso de diseño: "el diseñador, si bien no podrá prever todas las formas en que los productos complejos pueden ser utilizados, debe pensar en términos de posibilidades múltiples" [traducción de la autora] $\left(1995\right.$, p. 276) ${ }^{9}$.

8 "Before introducing something new, the designer must protect the status quo, which permits individuals to participate spontaneously and with little effort in the seductive immateriality of today's world".

9 "The designer can no longer foresee all the ways that complex products will be used and must think in terms of multiple possibilities rather than limited number of set functions". 
Como está expresado en el título del artículo, esta noción expande los límites del diseño. Incluye un nuevo concepto de producto y de usuario, y en consecuencia constituye una nueva relación entre ellos. El usuario forma parte del proceso de diseño y no es meramente su destinatario. Esto supone una visión integradora y superadora sobre la práctica del diseño: incluir en el proceso de diseñar una dimensión horizontal que se contrapone con el verticalismo de las miradas más clásicas de la modernidad, en la que la sutil diferencia reside en entender al usuario como coprotagonista del proceso y no como una voz a posteriori bajo la forma de feedback.

John Thackara, por su parte, enfoca el problema de la relación entre objeto, usuario y contexto desde la perspectiva de un diseño responsable. En su libro In the bubble. Designing in a complex world ${ }^{10}$, plantea una fuerte crítica hacia los modos modernos que convirtieron, durante la inauguración de la era industrial, a la tecnología en sinónimo de la subordinación de los intereses de la gente a los de ella, bajo las ideas de progreso y desarrollo continuo.

La idea de "que la gente se adapte" a las nuevas tecnologías nos afectó a todos. Creíamos que la línea de montaje y la estandarización iban a hacer del mundo un lugar mejor, pero de la mano de la eficiencia vino la deshumanización del trabajo [traducción de la autora]. (Thackara, 2005, p. 3) ${ }^{11}$

Aparece aquí la crítica recurrente a la promesa incumplida de la modernidad industrial, pero el autor no denosta la tecnología per se, sino que pretende instalar la pregunta cómo, es decir, la reflexión acerca de las consecuencias de lo que se diseña. Plantea la idea de un mundo con "menos cosas y más personas", con referencia a la instalación de un nuevo principio: el de ligereza, al cual deberá ceñirse el diseño donde el foco está puesto en los servicios y los sistemas, y no sobre las cosas. Se plantea aquí una nueva relación de consumo, basada en el diseño responsable y no en la tecnología.

10 Señalamos particularmente la idea de mundo "complejo", concepto central en el pensamiento posmoderno y cuyas implicancias atraviesan varios de los textos aquí trabajados.

11 "Getting people adapt" to new technology has affected us all. We believed that the assembly line and standardization would make the world a better place, yet along with efficiency came a dehumanization of work". 
En un mundo de menos cosas y más personas, vamos a seguir necesitando, sin embargo, sistemas, plataformas y servicios que le permitan a la gente interactuar más eficiente y placenteramente. Estas plataformas e infraestructuras van a requerir un poco de tecnología y mucho diseño [traducción de la autora]. (Thackara, 2005, p. 6) ${ }^{12}$

En este sentido, se trata de un planteo ético, inscrito en el discurso de la sustentabilidad, que habla de un mundo que drásticamente reducirá el volumen de hardware — cuya generación fuera la tarea tradicional del diseño-, por lo que cambia el eje de su función: el diseño como modo de pensamiento en oposición al que se dedicaba a hacer cosas.

Podemos afirmar, hasta aquí, que la dimensión inmaterial de la práctica del diseño aparece, con diversos matices, como una constante en los autores trabajados. La noción de discurso, ya visitada en Buchanan, está también presente en los trabajos del holandés Kees Dorst.

Dorst aborda las problemáticas de diseño como paradojas, en el sentido de que se encuentran estructuralmente constituidas por discursos en conflicto. Esta postura se encuentra en franca contraposición con el paradigma racional del problem-solving, el cual fue elaborado principalmente por H. A. Simon (1973), instalado como metodología y que, según Dorst, es aún una posición dominante en los discursos sobre el diseño.

El diseñador, en su situación problemática paradójica, necesita construir un diseño que trascienda o conecte los diferentes discursos, en sentido amplio (con la construcción de un metadiscurso), o simplemente en una instancia concreta del diseño para ser desarrollado. Para hacer esto, el diseñador debe sortear las formas de pensamiento encarnadas en los discursos. Este paso es probablemente incluir un fuerte elemento intuitivo. Basados en una comprensión clara de los discursos y las experiencias previas con situaciones paradójicas, se crea una solución que requiere ser evaluada desde el punto de vista de todos los diferentes discursos [...]. Para que la solución sea una solución, necesita estar reconocida como tal en los contextos de todos los discursos

12 "In a less-stuff-more-people world, we still need systems, platforms, and services that enable to interact more effectively and enjoyably. These platforms and infrastructures will require some technology and a lot of design". 
relevantes (en la práctica, esto a menudo significa, primero y principalmente, que debe ser aceptada por todos los stakeholders relevantes) [traducción de la autora]. (Dorst, 2006, p. 15) ${ }^{13}$

Mientras la noción de diseño como discurso es trabajada por Buchanan desde el aspecto retórico, Dorst se aproxima desde el concepto de la paradoja, el cual podríamos ligar a la teoría de la complejidad elaborada por Morin, en tanto lo paradojal consiste en operar con lógicas de sentido contrario sin excluirlas.

Un "problema de diseño" es tomado como una paradoja hecha de la colisión de discursos en conflicto. La naturaleza del diseño creativo reside en forjar las conexiones entre estos discursos, en un nivel general o en el diseño concreto [traducción de la autora]. (Dorst, 2006, p. 15) ${ }^{14}$

Nuestra argumentación se ha focalizado en el término "problema de diseño". El uso extendido de este término en los debates dentro y acerca del diseño lo convierten en uno de los términos básicos en la descripción metodológica de las actividades del diseño. Pero esperamos haber demostrado que este término es muy problemático en un contexto científico. [...] Por ahora, poner entre comillas el término "problemas de diseño" permite que emerjan nuevos marcos de referencia y descripciones de la actividad del diseño. De este ensayo surge una forma alternativa para describir al diseño como la resolución de paradojas entre discursos en una situación de diseño. Esta forma alternativa de describir al diseño echa nueva luz sobre su naturaleza y sobre la clase de

13 "The designer, in his/her paradoxical problematic situation, needs to construct a design that transcends or connects the different discourses, in a general sense (by the construction of a metadiscourse), or just in the concrete instance of the design-to-be-developed. To do this, the designer has to step out of the ways of thinking embodied in the discourses. This step is likely to include a strong intuitive element. Based upon a clear understanding of the discourses, and upon earlier experiences with paradoxical situations, a solution is created that needs to be evaluated from the standpoints of all the different discourses. [...] For the solution to be a solution, it needs to be recognized as such in the contexts of all relevant discourses. (In practice, this often means, first and foremost, that it should be acceptable to all the relevant stakeholders.)".

14 "A 'design problem' is taken as a paradox, made up out of the clash of conflicting discourses. The nature of creative design is the forging of connections between these discourses, on a general level or in the concrete design". 
creatividad que es parte y territorio del diseño [traducción de la autora]. (Dorst, 2006, p. 16) ${ }^{15}$

Simplemente, la idea de la resolución de paradojas entre discursos en una situación de diseño es una declaración superadora, en términos opuestos a la de cualquier descripción moderna. En primer lugar, el diseño como discurso, y, en segundo término, la resolución no de problemas de diseño, sino de paradojas en una situación de diseño, son parte, a su vez, de un discurso sobre la práctica renovado.

La concepción del diseño como un campo que excede la producción material de objetos, la expansión de sus áreas de alcance - a límites que aún no alcanzamos a vislumbrar-y el interés por incorporar el punto de vista del usuario en la reflexión sobre su práctica son partes del territorio que permite hablar de una "cultura del diseño". En este marco, Guy Julier intenta plantear la cultura del diseño como una disciplina propia de investigación que comprende tanto a los productos como a los consumidores.

En esa línea, advierte que con el capitalismo de mercado emerge una nueva generación de consumidores de diseño y se expanden de este modo sus competencias. Esto trae aparejado un desvanecimiento de los límites de las disciplinas tradicionales del diseño en virtud de que no se vende más un estilo visual, sino una forma de estructurar y gestionar el proceso de diseño.

La cultura del diseño es una "manera de hacer las cosas", que adopta un papel activo para transformar las prácticas de quienes están más allá de sus

15 'Our argument has focused on the term 'design problem'. The widespread use of this term in the vernacular discussions within and about design make it one of the basic terms in a methodological description of design activities. But we hope to have demonstrated that the term 'design problem' is very problematic in a scientific context. [...] Temporarily bracketing the term 'design problem' allows new frames of reference and descriptions of the design activity to emerge. Within this paper, that process has resulted in an alternative way to describe the design as the resolution of paradoxes between discourses in a design situation. This alternative way of describing design potentially sheds new light on the nature of design, and on the king of creativity that is part and parcel of design". 
administradores. Por tanto, entiende el contexto como algo circunstancial y no como algo dado: el mundo se puede cambiar mediante una nueva forma de cultura del diseño. (Julier, 2008/2010, p. 21)

Al igual que Moles, Julier tiene una visión del diseño que incluye a la inmaterialidad como tópico, entendiendo por diseño un concepto expandido que excede la prestación profesional para imbricarse con la cultura.Al respecto dice lo siguiente:

La cultura del diseño como objeto de estudio incluye, por tanto, los aspectos materiales e inmateriales de la vida cotidiana. Por una parte, se articula a través de imágenes, palabras, formas y espacios, pero, por otra, conjuga discursos, acciones, creencias, estructuras y relaciones. (Julier, 2008/2010, p. 23)

Y agrega:"la función del diseñador es la de crear valor. [...] Como consecuencia, surge un nuevo campo de actividades que orquestan y coordinan los procesos materiales y no materiales" (Julier, 2008/2010, p. 31).

Vemos en Julier una preocupación por instalar la cultura del diseño como marco teórico para el estudio del diseño, pero más interesante aún es su lectura del diseño, que se desplaza del eje de discusión moderna cuando deja de centrarse en los objetos: "la discusión ha dejado de centrarse en exclusiva en los objetos materiales, y se ha desplazado hacia una visión del diseño más integradora, que a su vez puede llegar a cuestionar el papel del diseñador" (Julier, 2008/2010, p. 77).

También recordamos el trabajo de Harold Nelson y Erik Stolterman, que en cierto modo integra todos los conceptos que estuvimos desarrollando con los autores precedentes en una teoría insertada en la cultura del diseño. En primer lugar, plantean que, para afrontar los desafíos propios de una realidad compleja, es necesaria una aproximación que trascienda los métodos tradicionales. Para ello, procuran desarrollar e implementar una cultura del diseño que entienden del siguiente modo:

La idea de cultura del diseño promueve una comprensión del diseño que trasciende contextos particulares, disciplinas específicas o conceptos individuales. Por ejemplo, es común la creencia de que el diseño es simplemente una forma de la creatividad. La creatividad es aquello que da al diseño una cualidad especial, pero aunque la creatividad es seminal para el diseño, este es más vasto 
y exhaustivo. El diseño incluye no solo pensamiento creativo sino actividad innovadora. Innovación difiere de la creatividad dado que se trata de una acción orientada, lograda a través de la manifestación e integración de conceptos creativos en el mundo real [traducción de la autora]. (Nelson y Stolterman, 2003 , p. 4$)^{16}$

Difieren de una visión moderna del diseño, que se aproxima a los métodos científicos, en el sentido de que el fin último sea el de crear objetos universales. Si bien la era tecnológica en la que vivimos ha cristalizado el método científico para describir la realidad, esto no va a servir para desarrollar una teoría del diseño.

No hay un abordaje científico para determinar algo concreto porque la ciencia es un proceso para discernir abstracciones que se aplican en categorías o en la taxonomía de un fenómeno, mientras que algo concreto es singular y único. Crear algo que es concreto y único, por lo tanto, no puede ser logrado usando un abordaje científico. [...] El resultado de un proceso de diseño específico, como un auto, un currículum o una estructura organizacional, es algo concreto y único, no son universales. [...] Incluso cuando los productos son diseñados a gran escala, con amplia distribución, aún poseen la cualidad de ser concretos y no universales, desde que no representan la única posibilidad de cumplir el mismo fin o servir el mismo propósito. (Nelson y Stolterman, 2003, p. 33) ${ }^{17}$

El objetivo de los autores no es el de desterrar la visión científico-racional del diseño, sino, por el contrario, sumar el carácter intuitivo como factor

16 "The idea of a design culture is one that promotes an understanding of design as transcendent of particular contexts, specific disciplines, or single concepts. For instance, it is commonly believed that design is simply a form of creativity. Creativity is thought of as the activity that gives design its special qualities. But, even though creativity is seminal to design, design is larger and more comprehensive. Design is inclusive not only of creative thinking but includes innovative activity as well. Innovation differs from creativity in that innovation is action oriented. It is achieved through the manifestation and integration of creative concepts into the real world".

17 "There is no scientific approach to determining the particular because science is a process of discerning abstractions that apply across categories or taxonomies of phenomena, while the particular, therefore, cannot be accomplished using a scientific approach. [...] The outcome of a specific design process, such as a car, a curriculum, or an organizational structure, is an ultimate particular. It is something unique. It is not the universal [...]. Even when products are designed in great numbers, with wide distribution, they still have the quality of being particular and not universal. 
constitutivo del diseño, al cual describen como un conocimiento complejo e integral.

En el mundo del diseño de hoy podemos encontrar abordajes modernos que reflejan las tradiciones científicas. [...] Estos abordajes no son los únicos que influencian cómo una sociedad y sus diseñadores adquieren conocimiento. [...] El diseño es considerado como un punto medio entre la intuición y la lógica, o la imaginación y la razón. (Nelson y Stolterman, 2003, pp. 36-37) 18 $^{18}$

Nelson y Stolterman, casi del mismo modo en que lo expresa Morin, critican el abordaje científico por su carácter reduccionista e introducen el término complejidad para referirse a la dinámica de las relaciones.

El abordaje analítico o científico para entender al mundo - a través de una observación y un análisis reduccionista - introdujo poderosas ideas, útiles para predecir y controlar la naturaleza por el bien de la humanidad. Sin embargo, estos beneficios también trajeron consecuencias negativas, ambas para la naturaleza y la humanidad.

La naturaleza no es una colección de elementos orgánicos e inorgánicos que existen aisladamente, como tampoco la humanidad es una colección de individuos aislados. Todo está relacionado con todo con grados de variabilidad e intensidad. Estas relaciones producen cualidades y atributos en múltiples niveles de resolución. La complejidad, un atributo característico derivado como consecuencia de la dinámica de interactividad de las relaciones, es la regla en el mundo real, mientras que la simplificación y el pensamiento reduccionista, tal como el de ignorar las relaciones y concomitancias emergentes, es una distracción peligrosa. (Nelson y Stolterman, 2003, p. 72-73) ${ }^{19}$

18 "In today's world of design, we can find modern approaches resembling all of these various scientific traditions. [...] These approaches, however, are not the only ones that can influence how a society and its designers acquire knowledge. [...] Design is also considered to be at a midpoint between intuition and logic, or imagination and reason".

19 "Scientific, or analytic, approaches to understanding the world - trough a consistent dedication to reductive observation and analysis - have provided powerful insights, helpful in predicting and controlling nature, for the betterment of humanity. But these gifts also have negative consequences, both to nature and to humans, as mentioned above.

Nature is not merely a collection of organic and inorganic elements or compounds, possessing attendant qualities and attributes, which exist in isolation. Nor is humanity merely a collection of individuals in 
Luego de haber expuesto esto, Nelson y Stolterman sostienen que un abordaje sistémico, es decir, que integre la complejidad, es necesario en el diseño; aún más, lo describen como su lógica.

Cuando vemos a la naturaleza y la actividad humana interrelacionada, estamos tomando un abordaje sistémico, el cual es opuesto a un abordaje reduccionista. Como diseñadores creemos que necesitamos ver al mundo desde la perspectiva sistémica. El abordaje sistémico es la lógica del diseño. (2003, p. 74 $)^{20}$

Como hemos dicho, describen la complejidad de manera muy similar a lo que podemos encontrar en Morin y la proponen como plataforma del diseño.

Complejidad se refiere a un proceso de inclusión abarcativo y completo de asimilación. [...] Es sobre tomar en consideración todas y cada una de las cosas potencialmente significativas. Es sobre mezclar manzanas con naranjas y hablar de repollos y reyes a través de un proceso que pone todo en relación y lo coloca en un contexto compartido. Esto se hace consistente e intencionalmente asegurando que toda situación de diseño sea apropiadamente compleja. (Nelson y Stolterman, 2003, pp. 85-86) ${ }^{21}$

Un diseño nunca existe aislado, siempre es parte de un todo y es en sí mismo un todo. En diseño, cuando decimos que algo es un todo nos referimos a

isolated proximity to one another. Everything is in relationship to everything else which varying levels of criticality and intensity. These relationships produce qualities and attributes at multiple levels of resolution. Complexity, a distinctive attribute arising as a consequence of the dynamic interactivity of relationships, is the rule in the real world, while simplification or reductionist thinking, such as ignoring relationships and concomitant emergent qualities, is a dangerous distraction".

20 "When we view nature and human activity as interrelated and interrelating, we are taking a systems approach, which is opposite to the reductionist approach described above. As designers, we believe that we need to view the world from this systems perspective. The systems approach is the logic of design".

21 "Complexity, or complexification, concerns the process of inclusion, encompassment, incorporation, assimilation and completeness. [...] It is about taking into consideration everything and anything that is judged to be potentially significant. It is a matter of 'mixing apples and oranges' and 'speaking of cabbages and kings' through the process of pulling everything into relationship and placing them in a shared context. This is done consistently and intentionally, assuring any design situation will be appropriately complex". 
que es un ensamble complejo de materia y substancia, significado y presencia. (Nelson y Stolterman, 2003, p. 117) 22

Siguiendo esta línea de pensamiento complejo, describen al proceso de diseño como una situación única, compleja e intransferible, y a diferencia de la idea de perfección, abogan por la de adecuación, sobre la cual encuadran al diseño. No hay una respuesta del diseño verdadera o falsa. El diseño es una forma única de pensamiento y acción.

Cada situación de diseño es compleja y única. Las soluciones de diseño que fueron creadas para una situación no encajan en otras. Por su puesto, se han trabajado ampliamente soluciones generales que se adaptan a cualquiera o a la mayoría de situaciones. Ellas no tienen la complejidad y el refinamiento o detalle suficiente para igualar la riqueza de una situación de diseño única. Esto no significa que los diseños deban ser complicados, costosos o excesivos. El mejor diseño para una situación debe ser elegantemente simple y económico, mientras que, al mismo tiempo, debe ser la respuesta más apropiada para los requerimientos únicos de una situación de diseño. (Nelson y Stolterman, 2003, p. 137) ${ }^{23}$

Esta idea es asimilable al planteo de Morin, quien establece una clara diferencia entre programa y estrategia: mientras que el primero implica una secuencia automática y preestablecida, una estrategia está destinada a modificarse en función de las circunstancias.

Diseñar es sobre manejar complejidad y riqueza, tensiones y contradicciones, posibilidades y límites; todo ello requiere un buen criterio. [...] El diseño

22 "A design never exists in isolation. It is always part of a larger whole and is itself whole. In design, when we say that something is a 'whole', it means that it is a complex ensemble of compound, meaning and presence".

23 "Every significant design situation is complex and unique. Design solutions that have been created for other complex, unique situations do not match the particular situation at hand. In addition, generalized solutions that fit all or most situations are couse and grossly formed. They do not have the complexity and refinement or detail sufficient to match the richness of a unique design situation. This does not mean that designs must be complicated, or expensive, or excessive in any other way as a consequence. The best design for a situation may be elegantly simple and economic, while at the same time being the most appropriate response to the unique requirements of the design situation". 
siempre ha sido, y seguirá siendo, colaborativo, aun cuando solo incluya a un diseñador y un cliente. Las actividades del diseño se han llevado adelante típicamente en grupos con muchos roles envueltos en relaciones complejas. [...] Es el diseñador individual el que tiene la responsabilidad de actuar sobre este camino para iniciar y desarrollar una cultura del diseño. (Nelson y Stolterman, 2003, p. 290) 24 $^{24}$

Baste volver al primer C. Jones y sus apreciaciones sobre simplicidad y trabajo en equipo, y compararlas con los conceptos de complejidad y creación colaborativa en Nelson y Stolterman para verificar la distancia conceptual de un discurso del diseño anclado en el pensamiento moderno frente a nuevas perspectivas de cuño posmoderno.

Perspectiva sistémica, tensiones y paradojas, complejidad e interrelación, singularidad antes que universalidad. Nunca antes el discurso sobre la práctica del diseño había empleado estos términos para definir su rol. El pensamiento posmoderno y la crítica a la razón universal que conlleva son ya parte del corpus teórico del diseño. El impacto de estas ideas en su práctica concreta, su enseñanza y su influencia en la cultura están aún por determinarse.

\section{CONSIDERACIONES FINALES. EL DISEÑO, UN CAMPO EN EXPANSIÓN}

Luego del recorrido hecho, sería ingenuo - y no es nuestra intenciónpretender caracterizar taxativamente los distintos discursos sobre la práctica del diseño como modernos o posmodernos. Entendemos que la cultura del diseño, como todo proceso cultural, comprende una simultaneidad de rasgos que, según la descripción de R. Williams, pueden ser residuales, dominantes o emergentes.

24 "Designing is about handling complexity and richness, tensions and contradictions, possibilities and limits, all of which require design to be a matter of making good judgement. [...] Design has always been, and will continue to be, collaborative at its core; even if that collaboration only includes one designer and one client. Design activities are tipically carried out in groups, with many roles involved in complex relationship. [...] It is the individual designer who has the responsibility to act in a design-driven way; to initiate and develop a design culture". 
Dentro del corpus de los discursos sobre la práctica del diseño, encontramos en convivencia aquellos que se podrían considerar modernos —algunos incluidos en este trabajo y otros tales como los de Frascara y Costa— junto a algunos que claramente no se alinean a los ejes dominantes.

Según podemos advertir en las lecturas que hemos revisado para este trabajo, hay un eje común que las atraviesa y que podríamos entender como un punto de inflexión entre un pensamiento moderno del diseño y otro más cercano a lo que algunos — por ejemplo, Lyotard, Vattimo, Huyssen, Jameson, Foster - entienden como posmoderno. Es la idea de que el diseño ya no tiene que ver con la materialización. Esta es la bisagra que, desde nuestro punto de vista, no solo amplía sus posibilidades, sino que cambia radicalmente la manera de pensarlo.

Siguiendo a Bourdieu, entendemos el diseño como un campo, por lo que su constitución y, eventualmente, sus transformaciones se dan a partir de la interacción entre los actores que pertenecen a él y su posicionamiento en la lucha de poder. Este es un proceso de legitimación que determinará el discurso dominante, pues entran en juego también posiciones emergentes. Es al interior de estas tensiones donde surge la transformación del discurso del diseño.

En este sentido, el diseño no se define a través de su medio - los objetos diseñados-, sino de su capacidad de pensarlos. Es aquí donde reside el aspecto liberado de la materialización de la cual hablábamos. Con diferentes matices, hemos visto esta perspectiva en autores como Moles, Nelson y Stolterman, Dorst, Thackara, Julier y Buchanan.

Abraham Moles (1995) dice, desde la noción de inmaterialidad y su consecuencia en la praxis del diseño, que "la actividad misma del diseño está cambiando porque las herramientas del diseñador se están volviendo inmateriales como las vidas de aquellos para quienes los productos se comercializan" (p. 270) ${ }^{25}$.

25 The design activity itself is changing because the designer's tools are becoming immaterial, as are the lives of those to whom the products are marketed. 
Harold Nelson y Erik Stolterman conciben al diseño como un modo de abordaje insertado en el marco de la complejidad:"diseñar es la habilidad de imaginar eso que aún no existe [...]. Diseñamos tanto nuestras cosmologías, hogares, negocios y vidas como artefactos materiales" $(2003 \text {, p. } 10)^{26}$.

Richard Buchanan entiende al diseño desde lo discursivo: "el diseñador, en vez de simplemente hacer un objeto o cosa, está en realidad creando un argumento persuasivo" [traducción de la autora] (1989, pp. 95-96) ${ }^{27}$.

Kees Dorst incorpora el concepto de paradoja a partir de la formulación del diseño como discurso de Buchanan:

De este ensayo surge una forma alternativa para describir al diseño como la resolución de paradojas entre discursos en una situación de diseño. Esta forma alternativa de describir el diseño echa nueva luz sobre su naturaleza y sobre la clase de creatividad que es parte y territorio del diseño. [traducción de la autora] $(2006, \text { p. 17) })^{28}$

John Thackara, desde una perspectiva ética anclada en la noción de sustentabilidad, dice lo siguiente:

En un mundo de menos cosas y más personas, vamos a seguir necesitando, sin embargo, sistemas, plataformas y servicios que le permitan a la gente interactuar más eficiente y placenteramente. Estas plataformas e infraestructuras van a requerir un poco de tecnología y mucho diseño. [traducción de la autora] $(2005, \text { p. } 6)^{29}$

26 Design is the ability to imagine that-which-does-not-yet-exist. [...] We design our cosmologies, our homes, our businesses and our lives, as well as our material artifacts.

27 The designer, instead of simply making an object or thing, is actually creating a persuasive argument.

28 Within this paper, that process has resulted in an alternative way to describe the design as the resolution of paradoxes between discourses in a design situation. This alternative way of describing design potentially sheds new light on the nature of design, and on the kind of creativity that is part and parcel of design.

29 In a less-stuff-more-people world, we still need systems platforms, and services that enable people to interact more effectively and enjoyably. These platforms and infrastructures will require some technology and a lot of design. 
Guy Julier (2010) hace énfasis en la cultura del diseño:

La forma en que un producto se presenta ante su público — como un objeto de diseño - se convierte en su valor primigenio, [...] como afirman Lash y Urry: lo que se produce cada vez más no son objetos materiales, sino signos. (p. 65)

Al desplazar la materialidad como eje vertebral del diseño, se abren nuevos horizontes para su práctica y por ello dejamos planteada la idea de campo expandido. Si bien se podría señalar que una idea del diseño, no como ente materializador de objetos, sino a partir de su carácter transformador y de intervención cultural, ya estaba presente en defensores del proyecto moderno como O. Aicher, esta se da siempre a partir de la materialización de elementos concretos y de pensar que estos elementos intervienen en la cultura. En cambio, los autores que recorren líneas de pensamiento más afines a la posmodernidad asignan al diseño el carácter de sistema de pensamiento en tanto constitutivo de la cultura, desde un abordaje complejo e independiente de la construcción de objetos.

Reconocidos diseñadores e intelectuales como Bill Moggridge, David Kelley, John Maeda y Bruce Mau, entre otros, operan hoy en las fronteras - y más allá- de lo que el movimiento moderno definió como diseño.Aun considerando el carácter extendido de ciertos neologismos, es innegable que conceptos como diseño de experiencias serían impensables en un marco estrictamente moderno del discurso del diseño.

Lo descrito a lo largo de este trabajo da apenas una idea general del estado de la cuestión de los nuevos discursos sobre la práctica del diseño, y nuestra hipótesis se encuentra en una fase embrionaria, pero creemos que cumple el propósito de dejar planteado un posible camino para indagaciones posteriores sobre la base de las interrogantes que no han sido respondidas o de las que pueden ser formuladas.

Determinar si estos nuevos discursos dan cuenta de una expansión del campo del diseño tal y como hemos desarrollado hasta aquí o si bien son indicio de una transformación mucho más profunda y constitutiva, capaz de poner en cuestión la noción misma de diseño, es una de las preguntas que quedan planteadas. 


\section{REFERENCIAS}

Baudrillard, J. (1997). Crítica de la economía política del signo. Buenos Aires: Siglo XXI. (Trabajo originalmente publicado en 1974)

Bourdieu, P. (2005). Las reglas del arte. Barcelona: Anagrama.

Buchanan, R. (1989). Declaration by design: rhetoric, argument, and demostration in design practice. En V. Margolin (Ed.), Design discourse. History. Theory. Criticism (pp. 91-109). Chicago: University Of Chicago Press. (Trabajo originalmente publicado en 1985)

Buchanan, R. (2010). Branzi's dilemma: design in contemporary culture. En R. Buchanan, V. Margolin y D. Doordan (Eds.), The designed world: images, objects, environments (pp. 13-27). Nueva York: Berg Publishers. (Trabajo originalmente publicado en 1994)

Buchanan, R., y Margolin, V. (1995). Discovering design: explorations in design studies. Chicago: University Of Chicago Press.

Devalle,V. (2009a). La travesía de la forma. Buenos Aires: Paidós.

Devalle, V. (2009b). El análisis cultural. Nuevas perspectivas para pensar el diseño. En L. Arfuch y V. Devalle (comp.), Visualidades sin fin. Imagen y diseño en la sociedad global (pp. 41-71). Buenos Aires: Prometeo.

Dorst, K. (2006). Design problems. Design paradoxes. Design Issues, 22(3), $4-17$.

Habermas, J. (2008). La modernidad, un proyecto incompleto. En H. Foster (Ed.), La posmodernidad (pp. 19-36). Barcelona: Kairós.

Jones, C. (1976). Métodos de diseño. Barcelona: Gustavo Gili.

Julier, G. (2010). Cultura del diseño. Barcelona: Gustavo Gili.

Krauss, R. (2008). La escultura en el campo expandido. En H. Foster (Ed.), La posmodernidad (pp. 59-74). Barcelona: Kairós.

Lyotard, J. F. (1993). La condición posmoderna. Buenos Aires: Planeta Agostini. (Trabajo originalmente publicado en 1979) 
Lyotard, J. F. (1994). La posmodernidad. (Explicada a los niños). Barcelona: Gedisa. (Trabajo originalmente publicado en 1986)

Maldonado, T. (1993). Diseño industrial reconsiderado. Barcelona: Gustavo Gili. (Trabajo originalmente publicado en 1977)

Maldonado, T. (octubre de 1997). Proyectar hoy. Revista Contextos, 1(1), $54-58$.

Moles, A. (1973). El kitsch. El arte de la felicidad. Buenos Aires: Paidós. (Trabajo originalmente publicado en 1971)

Moles, A., (1995). Design and immateriality: what of it in a post industrial society? En V. Margolin y R. Buchanan (Eds.), The idea of design (pp. 268-274). Massachusetts:The MIT Press.

Morin, E. (1997). Introducción al pensamiento complejo. Buenos Aires: Gedisa. (Trabajo originalmente publicado en 1990)

Nelson, H., y Stolterman, E. (2003). The design way. Intentional change in an unpredictable world. Nueva Jersey: Educational Technology Publications Englewood Cliffs.

Pelta, R. (2004). Diseñar hoy. Temas contemporáneos de diseño gráfico. Buenos Aires: Paidós.

Thackara,J. (2005). In the bubble. Designing in a complex world. Cambridge: The MIT Press.

\section{BIBLIOGRAFÍA}

Aicher, O. (1994). El mundo como proyecto. Barcelona: Gustavo Gili.

Alexander, C. (1971). Ensayo sobre la síntesis de la forma. Buenos Aires: Infinito.

Berman, M. (2001). Todo lo sólido se desvanece en el aire. La experiencia de la modernidad. Buenos Aires: Siglo XXI. 
Berman, M. (abril-mayo 2005). El camino ancho y abierto. Revista Tipográfica, (65), 24-27.

Bonsiepe, G. (1975). Diseño industrial: artefacto y proyecto. Madrid:Alberto Corazón.

Bonsiepe, G. (1999). Del objeto a la interfase. Mutaciones del diseño. Buenos Aires: Infinito.

Buchanan, R., y Margolin, V. (1989). The idea of design. Massachusetts: The MIT Press.

Buchanan, R., Doordan, D., y Margolin, V. (2010). The designed world: images, objects, environments. Nueva York: Berg Publishers.

Bürdek, B. (2002). Diseño. Historia, teoría y práctica del diseño industrial. Barcelona: Gustavo Gili.

Casullo, N. (comp.). (2004). El debate modernidad postmodernidad [edición ampliada]. Buenos Aires: Retórica.

Dorst, K. (1997). Describing design. A comparison of paradigms. Rotterdam: Vormgeving.

Foster, H. (2008). Introducción al posmodernismo. En H. Foster (Ed.), La posmodernidad (pp. 7-17). Barcelona: Kairós.

Foucault, M. (2008). Las palabras y las cosas. Una arqueología de las ciencias humanas. Buenos Aires: Siglo XXI.

Jameson, F. (2008). Lo utópico, el cambio y lo histórico en la posmodernidad. En N. Casullo (comp.), El debate modernidad postmodernidad (pp. 269-277). Buenos Aires: Retórica.

Lawson, B. (2006). How designers think. The design process demystified. Oxford: Architectural Press.

Lyotard, J. F. (2008). Qué era la posmodernidad. En N. Casullo (comp.), El debate modernidad postmodernidad (pp. 65-73). Buenos Aires: Retórica. 
Maldonado, T. (2004). El movimiento moderno y la cuestión post. En N. Casullo (comp.), El debate modernidad postmodernidad (pp.259-265). Buenos Aires: Retórica.

Maldonado, T. (2004). ¿Es la arquitectura un texto?Y otros escritos. Buenos Aires: Infinito.

Margolin, V. (1989). Expanding the boundaries of design: the product environment and the new user. En R. Buchanan yV. Margolin, The idea of design (pp. 275-282). Massachusetts:The MIT Press.1996

Meggs, P. (2000). Historia del diseño gráfico. México D. F: McGraw-Hill.

Pevsner, N. (1972). Pioneros del diseño moderno. De Williams Morris a Walter Gropius. Buenos Aires: Infinito.

Press, M., y Cooper, R. (2009). El diseño como experiencia. El papel del diseño y los diseñadores en el siglo XxI. Barcelona: Gustavo Gili.

Thackara, J. (1988). Design after modernism: beyond the object. Nueva York: Thames \& Hudson.

Touraine, A. (1994). Crítica de la modernidad. Buenos Aires: FCE.

Vattimo, G. (2000). El fin de la modernidad. Nihilismo y hermenéutica en la cultura posmoderna. Barcelona: Gedisa.

Venturi, R. (2012). Complejidad y contradicción en la arquitectura. Barcelona: Gustavo Gili.

Williams, R. (1994). Sociología de la cultura. Buenos Aires: Paidós.

Williams, R. (2000). Marxismo y literatura. Barcelona: Península. 
